\title{
Alterations in Effective Connectivity of the Hippocampus in Migraine without Aura
}

\author{
Yadi Zhu ${ }^{1,2, *}$ \\ Lingling Dai ${ }^{1,2, *}$ \\ Hongru Zhao ${ }^{3, *}$ \\ Boan $\mathrm{Ji}^{4}$ \\ Yang $\mathrm{Yu}^{1,2}$ \\ Hui Dai $\mathbb{D}^{1,2}$ \\ Chunhong $\mathrm{Hu}^{1,2}$ \\ Ximing Wang ${ }^{1,2}$ \\ Jun $\mathrm{Ke} \mathbb{D}^{1,2}$
}

'Department of Radiology, The First Affiliated Hospital of Soochow University, Soochow, Jiangsu Province, People's Republic of China; ${ }^{2}$ Institute of Medical Imaging, Soochow University, Soochow, jiangsu Province, People's Republic of China; ${ }^{3}$ Department of Neurology, The First Affiliated Hospital of Soochow University, Soochow, Jiangsu Province, People's Republic of China; ${ }^{4}$ Medical School of Soochow University, Soochow, Jiangsu Province, People's Republic of China

*These authors contributed equally to this work

\begin{abstract}
Purpose: Neuroimaging studies on migraine have revealed structural and functional alterations in the hippocampus, a region involved in pain processing and stress response. This study was designed to investigate whether effective connectivity of this region is disrupted in migraine and relates to chronicity of this disease.
\end{abstract}

Patients and Methods: In 39 episodic migraine (EM) patients, 17 chronic migraine (CM) patients, and 35 healthy controls, we investigated differences in the directional influences between the hippocampus and the rest of the brain by combining resting-state functional magnetic resonance imaging and Granger causality analysis (GCA), with bilateral hippocampus as seed regions. The associations between directional influences and the clinical variables were also examined.

Results: Comparing each patient group to the control group, we found increased and decreased negative influence on the hippocampus exerted by the bilateral visual areas and right dorsolateral prefrontal cortex (dIPFC), respectively. The hippocampus showed increased positive influence on the right posterior insula and medial prefrontal cortex (mPFC), as well as increased negative influence on the left cerebellum in $\mathrm{CM}$ patients relative to EM patients and healthy controls. Furthermore, across all patients, the migraine frequency exhibited a positive and negative association with causal influence from the hippocampus to mPFC and left cerebellum, respectively.

Conclusion: Migraine patients have abnormal effective connectivity between the hippocampus and multiple brain regions involved in the sensory and cognitive processing of pain. Disrupted directional influences to the hippocampus exerted by dIPFC and bilateral visual areas were common features of EM and CM patients. Directional influences from the hippocampus to $\mathrm{mPFC}$ and left cerebellum may be useful imaging biomarkers for assessing migraine frequency.

Keywords: migraine, hippocampus, effective connectivity, resting-state, functional magnetic resonance imaging

\section{Introduction}

Migraine is a common and debilitating neurological disorder characterized by attacks of recurrent, unilateral, moderate or severe, throbbing, and pulsating headaches, which are often accompanied by sensory hypersensitivity, as well as cognitive and emotional dysfunction. ${ }^{1}$ With a 1 -year prevalence of $12 \%$ in the general population, ${ }^{2}$ migraine has been identified as the second most disabling condition worldwide ${ }^{3}$ for its substantial impact on physical, social, academic, and occupational functioning of patients. ${ }^{4}$ So far, however, the pathophysiology of migraine has not been fully elucidated.
Correspondence: Ximing Wang; Jun Ke Department of Radiology, The First Affiliated Hospital of Soochow University, No. 188 Shizi Road, Soochow, 215000, Jiangsu Province, People's Republic of China

Tel +86-5 I2-67973062

Fax +86-5 I 2-6797306I

Email wangximing1998@।63.com;

fmrikej@|26.com 
In the past decades, important progress has been made in the understanding of the neural correlate of migraine by applying advanced neuroimaging techniques. Structural and regional brain activity alterations have been revealed in multiple brain areas important for the sensory, affective, and cognitive components of pain processing, such as the somatosensory cortex, insula, amygdala, medial prefrontal cortex (mPFC) and dorsolateral prefrontal cortex (dlPFC) ${ }^{5,6}$ Recent studies support migraine as a complex brain network disorder, involving integrated activity across cortical and subcortical brain areas. ${ }^{7}$ Resting-state functional magnetic resonance imaging (rs-fMRI), independent of tasks or stimulations, serves as a useful tool for investigating the functional organization in the brains of patients with migraine. Utilizing seed-based functional connectivity (FC) analysis, abnormal resting-state FC with the amygdala, insula, periaqueductal gray matter, hypothalamus, and basal ganglia has been detected in prior research on migraine. ${ }^{8}$ In studies using independent component analysis, migraine patients have been found to present atypical FC within and between several resting state networks, including the default mode network (DMN), central executive network and salience network. ${ }^{6}$

An additional brain structure that has garnered increased attention as an important contributor to the pathophysiology of migraine is the hippocampus. As a component of the limbic system and DMN, ${ }^{9}$ the hippocampus is well known for its essential role in learning and memory. It also participates in pain processing, painrelated attention and anxiety, ${ }^{10}$ as well as stress response. ${ }^{11}$ Accumulating evidence has implicated the role of a maladaptive stress response in the mechanism of migraine, especially in chronic migraine. ${ }^{12}$ Taken together, it is reasonable that symptoms of migraine may be associated with disrupted hippocampal structure and function. Indeed, altered volume of the hippocampus has been repeatedly detected, ${ }^{13,14}$ though the directions of change were not completely consistent. The inconsistency may be partially due to the mixing of episodic migraine (EM) and chronic migraine (CM) into the same patient group. A longitudinal study on newly diagnosed EM discovered increased migraine frequency at follow-up relative to baseline, which was accompanied by decreased hippocampal volume. ${ }^{15}$ Liu et al. found that EM patients carrying a COMT Val homozygous had a larger hippocampus compared with controls with the same polymorphism. ${ }^{16}$ In one previous study, we found the hippocampus volume of the healthy control (HC) group was smaller than that of the
EM group, but was larger than that of the CM group. ${ }^{17}$ This finding accorded with two cross-sectional studies suggesting an adaptive volume increase at low headache frequency and a maladaptive volume decrease at higher headache frequency, and proved the necessity to compare migraine patients of different headache frequency when investigating alterations in hippocampus-related brain circuit.

With respect to the hippocampal brain connectivity, Gao et al. demonstrated decreased intraregional FC in EM patients by calculating local functional connectivity density during resting state. ${ }^{18}$ However, the local connectivity reflects a local hub and represents regional energy demand, ${ }^{19}$ indicating it could serve as a marker of brain activity rather than that of functional interactions between brain areas. For remote connectivity, reduced hippocampal FC was reported in high frequency versus low frequency migraine patients in the temporal lobe, insula, right amygdala, and middle frontal cortex during thermal stimulation. ${ }^{20}$ At resting state, Wei et al. showed that the hippocampus had decreased FC with the supplementary motor area and inferior parietal lobe in migraine patients compared with healthy controls. ${ }^{21}$ Recently, a study focusing on the reward system revealed weaker FC between the right nucleus accumbens and hippocampus in patients with $\mathrm{EM}^{22}$ In one study examining the contribution of genotype to hippocampal FC changes in female patients with EM, Liu et al. found no significant interaction effect for disease and catechol-O-methyltransferase genotype, but reported decreased FC between hippocampus and multiple brain regions including the $\mathrm{mPFC}$, insula and amygdala in EM patients with val homozygote. ${ }^{16}$ Chong et al. found stronger structural covariance between the hippocampus and corticolimbic regions in migraine. ${ }^{13}$ However, the direction of influence of the hippocampus on other structures, which may provide novel insight into the neural basis of migraine, could not be delineated in the above-mentioned studies on brain connectivity. Moreover, it remains to be determined whether the directed influence (effective connectivity, EC) of the hippocampus is different between patients with $\mathrm{CM}$ and EM.

We therefore sought to investigate the effect of migraine on the EC of the hippocampus with the rest of the brain by employing rs-fMRI and Granger causality analysis (GCA). EC differences between patients with EM and CM were also compared to determine whether this measure was associated with migraine subtypes and migraine frequency. Based on previous findings on migraine, we hypothesized migraine 
patients would present EC abnormalities in regions involved in pain processing, such as the insula and prefrontal cortex. Moreover, EC of the hippocampus may differ in patients with different migraine types, and EC alterations may be correlated with clinical symptoms of migraine such as migraine frequency.

\section{Methods}

\section{Participants and Clinical Assessment}

A total of 91 individuals, including 56 patients with migraine without aura and 35 healthy controls, were enrolled in this case control study. Participants were recruited from the Department of Neurology at the first affiliated hospital of Soochow University. All subjects gave written informed consent prior to participation. The study was carried out in accordance with the Declaration of Helsinki, and was approved by the ethics committee of the first affiliated hospital of Soochow University (Approval no. 2021246). Diagnoses of migraine were made by an experienced neurologist according to the criteria established in the International Classification of Headache Disorders (ICHD-3 beta) guidelines. ${ }^{1}$ Thirtynine patients were diagnosed with EM, and 17 patients were diagnosed with CM. Headache frequency was calculated as the average number of headache days per month. According to the headache frequency, the EM group was further divided into infrequent episodic migraine (IEM, with $\leq 2$ headache days per month) and frequent episodic migraine (FEM, with 3 to 14 headache days per month) subgroups. Other assessed clinical indices included disease duration (years with migraine), and pain intensity which was rated using a 10-point visual analog scale (VAS) from 0 (none) to 10 (very severe). All migraine patients had not taken any migraine preventive medication for at least 3 months prior to the study. Patients were free of migraine attacks for at least 72 hours before testing, during or on the day following the MRI scan. ${ }^{23}$ For the HC group, subjects never had any primary headache disorders or other types of headache, and no family history of migraine was also required. General exclusion criteria for migraine patients and controls included age $<18$ years or $>65$ years; left-handedness; history of neurological or psychiatric disorders; cardiovascular disease or trauma; metabolic disorders; medication overuse headache; other pain conditions; drug or alcohol abuse; MRI contraindications; and excessive movement during the MRI scanning (translation $>1.5 \mathrm{~mm}$ or rotation $>1.5^{\circ}$ at any direction).

\section{MRI Data Acquisition}

MRI scans were performed on a 3.0 Tesla scanning system (MAGNETOM Skyra, Siemens Healthcare, Erlangen, Germany) at the Department of Radiology, the first affiliated hospital of Soochow University. The axial resting-state functional images were collected using an echoplanar imaging sequence with the following parameters: $\mathrm{TR}=2000 \mathrm{~ms}, \mathrm{TE}=30 \mathrm{~ms}$, flip angle $=90^{\circ}, \mathrm{FOV}=$ $256 \times 256 \mathrm{~mm}^{2}$, matrix $=64 \times 64$, slice number $=33$, slice thickness $=4 \mathrm{~mm}$, no intersection gap, total volume number $=240$. The sections were placed approximately parallel to the anterior commissure-posterior commissure line. High-resolution T1-weighted images were acquired using a sagittal fast spoiled gradient recalled echo sequence with the following parameters: $\mathrm{TR}=2300 \mathrm{~ms}, \mathrm{TE}=2.98 \mathrm{~ms}$, matrix $=256 \times 256, \mathrm{FOV}=256 \times 256 \mathrm{~mm}^{2}$, slice thickness $=1 \mathrm{~mm}$. During the rs-fMRI scans, participants were instructed to lie still with their eyes closed, but not to fall asleep or think about anything in particular. The structural images were examined by two professional radiologists to exclude the possibility of clinically silent lesions.

\section{Data Preprocessing}

The imaging data were preprocessed using Statistical Parametric Mapping (SPM12, http://www.fil.ion.ucl.ac.

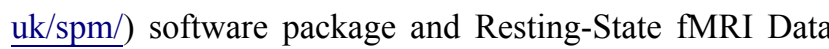
Analysis Toolkit software (REST, http://www.restfmri. net). The first 10 time points of the functional data were discarded to reduce the effect of instability magnetization at the initial scans, leaving 230 time points for further processing. The remaining images were slice-time corrected, realigned, and co-registered with the individual $\mathrm{T} 1-$ weighted images. The co-registered structural images were then segmented into gray matter, white matter (WM), and cerebrospinal fluid (CSF), and spatially normalized into standard Montreal Neurological Institute (MNI) space with a final size of $3 \times 3 \times 3 \mathrm{~mm}^{3}$. The resulting normalization matrix was then applied to the functional images. After that, linear drift was removed and a band-pass frequency filter $(0.01-0.08 \mathrm{~Hz})$ was used to reduce low-frequency drift and physiological high frequency noise. The obtained images were then spatially smoothed using an 8-mm fullwidth half maximum, isotropic Gaussian kernel. To further reduce the influence of confounding factors, nine nuisance variants, including six head motion parameters, time series predictors for global signal, WM signal and CSF signal were regressed from the time series. 


\section{Voxel-Wise GCA}

The aim of the current study was to examine the EC between the hippocampus and the rest of the brain. Therefore, the left and right hippocampus in the automated anatomical labeling (AAL) template were set as the regions of interest. Bivariate first-order coefficient-based voxel-wise GCA was then performed within the gray matter mask using the REST software. Granger causality evaluates the causal effect of the seed region on every other voxel in the brain ( $\mathrm{X}$ to $\mathrm{Y}$ effect), as well as the causal effect of every voxel in the brain on the seed region ( $\mathrm{Y}$ to $\mathrm{X}$ effect). A positive coefficient from $\mathrm{X}$ to $\mathrm{Y}$ indicates that activity in region $\mathrm{X}$ exerts a causal influence on the activity of region $\mathrm{Y}$ in the same direction (i.e. positive or excitatory influence). Similarly, a negative coefficient from $\mathrm{X}$ to $\mathrm{Y}$ suggests that activity of region $\mathrm{X}$ predicts the subsequent activity in region $\mathrm{Y}$ in the opposite direction (i.e. negative or inhibitory influence). ${ }^{24}$ For further statistical analysis, Fisher transform was applied to the resulting coefficient to produce $\mathrm{Z}$-score maps.

\section{Statistical Analysis}

Chi-squared test was performed to analyze gender distribution, and one-way analysis of variance (ANOVA) was conducted for age and education level. For clinical variable, independent $t$-test was utilized to examine differences between the patient groups. The above analyses were conducted with SPSS version 16.0 (SPSS Inc., Chicago, IL, USA), and the significance threshold was set at $\mathrm{P}<0.05$.

The Granger causality maps of the three groups were compared using ANOVA in SPM8 with age, gender, and education level as covariates, followed by post hoc $t$-tests to detect between-group differences. For post hoc $t$-tests, a mask based on significant clusters of the $F$-test was used. To correct for multiple comparison in the ANOVA and post hoc $t$-tests for Granger causality maps, a threshold adjustment based on Gaussian random field theory (voxel-level $\mathrm{P}<0.01$, cluster level $\mathrm{P}<0.05$, two tailed) was applied within the gray matter mask using the Data Processing and
Analysis for Brain Imaging (DPABI, http://rfmri.org/dpabi) package. To explore whether Granger causality of the hippocampus was impacted by headache frequency in the EM group, independent two-sample $t$-test was further conducted for the IEM and FEM subgroups within a mask derived from the results of the ANOVA mentioned above and was corrected for multiple comparison using the same approach.

For brain regions showing significant group differences, Granger causality coefficients were extracted to investigate the directions of the causal influences between hippocampus and these regions in each group separately. Pearson correlation analysis was then performed to explore the association between the mean Granger causality coefficient within each region and clinical indices, such as the migraine frequency and pain intensity. The correlation analyses were accomplished using SPSS 16.0, with a significant threshold of $\mathrm{P}<0.05$ (not corrected).

\section{Results}

\section{Demographics and Clinical Data}

Demographic and clinical characteristics of the EM patients, CM patients, and healthy controls were given in Table 1. No significant difference was found in gender distribution $(\mathrm{P}=0.06)$ and years of education $(\mathrm{F}=$ $1.663, \mathrm{P}=0.195)$ among the three groups. The age of the $\mathrm{CM}$ group was significantly higher than that of the EM and $\mathrm{HC}$ groups $(\mathrm{F}=8.627, \mathrm{P}<0.001)$. The $\mathrm{CM}$ group showed a higher migraine frequency than the EM group $(\mathrm{P}<0.001)$. There was no significant difference in pain intensity between the two migraine groups $(\mathrm{P}=0.635)$.

\section{Causal Influence from Seed Region to Whole Brain}

There were significant differences in causal flow from the left hippocampus to mPFC (Table 2, Figure 1A). Post hoc $t$-test revealed that the $\mathrm{CM}$ group showed increased positive influence from the left hippocampus to $\mathrm{mPFC}$ compared with the

Table I Demographic and Clinical Characteristics of Patients and Healthy Controls

\begin{tabular}{|l|l|l|l|l|l|l|}
\hline & Male/Female & Age (Year) & Education (Year) & Day with Headache $^{\mathrm{a}}$ & Disease Duration (Year) $^{\text {VAS }^{\mathbf{b}}}$ \\
\hline EM $(\mathrm{n}=39)$ & $9 / 30$ & $39.74 \pm 11.59$ & $10.33 \pm 4.02$ & $3.75 \pm 2.64$ & $14.63 \pm 7.15$ & $6.22 \pm 1.77$ \\
$\mathrm{CM}(\mathrm{n}=17)$ & $9 / 8$ & $49.59 \pm 14.64$ & $9.71 \pm 3.94$ & $19.56 \pm 4.17$ & $19.53 \pm 13.24$ & $7.24 \pm 1.89$ \\
$\mathrm{HC}(\mathrm{n}=35)$ & $15 / 20$ & $34.91 \pm 10.89$ & $11.80 \pm 4.92$ & & & $0.078^{\mathrm{e}}$ \\
$\mathrm{P}$ value & $0.060^{\mathrm{c}}$ & $<0.001^{\mathrm{d}}$ & $0.195^{\mathrm{d}}$ & $<0.001^{\mathrm{e}}$ & $0.635^{\mathrm{e}}$ \\
\hline
\end{tabular}

Notes: Continuous variables are given as mean \pm standard deviation. ${ }^{\mathrm{a} D a y s}$ of migraine per month; ${ }^{\mathrm{b}}$ Visual analog scales, grading pain severity on a scale of I to I0; ${ }^{\mathrm{P}} \mathrm{P}$ value obtained with Chi-square test; ${ }^{\mathrm{d}} \mathrm{P}$ value obtained with one-way analysis of variance; ${ }^{\mathrm{e}} \mathrm{P}$ value obtained with independent $t$-test.

Abbreviations: EM, the episodic migraine group; CM, the chronic migraine group; HC, the healthy control group. 
Table 2 Regions Showing Significant Difference in Hippocampus Effective Connectivity Among Groups

\begin{tabular}{|c|c|c|c|c|c|c|c|}
\hline \multirow[t]{2}{*}{ Brain Region } & \multirow[t]{2}{*}{ Hemi } & \multirow[t]{2}{*}{ Voxel } & \multirow[t]{2}{*}{ MNI Coordinate $(x, y, z)$} & \multicolumn{3}{|c|}{ GC Coefficient } & \multirow[t]{2}{*}{ Peak F Score } \\
\hline & & & & CM & EM & HC & \\
\hline \multicolumn{8}{|c|}{ Causal influence from left hippocampus to whole brain } \\
\hline mPFC & $L / R$ & 288 & $6,54,21$ & 0.86 & -1.08 & -0.57 & 13.92 \\
\hline \multicolumn{8}{|c|}{ Causal influence from whole brain to left hippocampus } \\
\hline Visual areas & L & 353 & $-24,-78,21$ & -0.49 & -0.38 & 0.13 & 9.67 \\
\hline Visual areas & $\mathrm{R}$ & 136 & $21,-54,12$ & -0.60 & -0.18 & 0.18 & 12.01 \\
\hline MFG/SFG & $\mathrm{R}$ & 80 & $27,54,18$ & -0.31 & -0.32 & -0.64 & 9.46 \\
\hline \multicolumn{8}{|c|}{ Causal influence from right hippocampus to whole brain } \\
\hline Insula & $\mathrm{R}$ & 77 & $39,-12,-3$ & 0.39 & -0.18 & -0.49 & 8.6 \\
\hline Cerebellum & $\mathrm{L} / \mathrm{R}$ & 97 & $-18,-63,-24$ & -1.16 & 0.40 & -0.03 & 9.56 \\
\hline \multicolumn{8}{|c|}{ Causal influence from whole brain to left hippocampus } \\
\hline Visual areas & L & 154 & $-15,-78,3$ & -0.29 & -0.14 & 0.44 & 9.32 \\
\hline Visual areas & $\mathrm{R}$ & 57 & $15,-84,-9$ & -0.35 & -0.01 & 0.29 & 9.02 \\
\hline
\end{tabular}

Abbreviations: Hemi, hemisphere; L, left; R, right; MNI, Montreal Neurological Institute; GC, Granger causality; EM, the episodic migraine group; CM, the chronic migraine group; HC, the healthy control group; mPFC, medial prefrontal cortex; MFG, middle frontal gyrus; SFG, superior frontal gyrus.

EM and HC groups (Figure 2A, Supplementary Table 1). The $\mathrm{CM}$ patients exerted a positive influence from the left hippocampus to $\mathrm{mPFC}$, while the EM patients and healthy controls showed negative influence (Figure 1A). No significant difference in causal flow from the left hippocampus to $\mathrm{mPFC}$ was found between the EM and HC groups. Additionally, the FEM subgroup showed decreased negative influence from the left hippocampus to mPFC compared with the IEM subgroup.

Analyses for the right hippocampus revealed significant differences in causal flow from this region to the left cerebellum and right posterior insula (Table 2, Figure 1B). Post hoc $t$-test demonstrated that the CM group showed increased positive influence to the right posterior insula and increased negative influence to the left cerebellum when compared with the other two groups (Figure 3A, Supplementary Table 2). The right hippocampus showed positive influence to the right posterior insula in the $\mathrm{CM}$ group but negative influence in the other two groups (Figure 1B). As for the left cerebellum, CM patients exerted negative influence from right hippocampus to this region, while EM patients and healthy controls showed positive and negative influence, respectively (Figure 1B). No significant difference in causal flow from the right hippocampus to the right posterior insula or left cerebellum was detected between the EM and HC groups.

\section{Causal Influence from Whole Brain to Seed Region}

There were significant differences in causal flow from the right middle frontal gyrus/superior frontal gyrus (MFG/ SFG) to the left hippocampus, and from bilateral visual areas to the left hippocampus (Table 2, Figure 1C) and right hippocampus (Table 2, Figure 1D). Compared with healthy controls, both $\mathrm{CM}$ patients and EM patients showed decreased negative influence from the right MFG/SFG to the left hippocampus (Figure 2B, Supplementary Table 3), and increased negative influence from bilateral visual areas to the bilateral hippocampus (Figures 2B and 3B, Supplementary Tables 3 and 4). In each group, the right MFG/SFG showed negative influence to the left hippocampus (Figure 1C). As for the visual areas, negative and positive influences from this region to the bilateral hippocampus were detected in $\mathrm{CM}$ patients and healthy controls, respectively, while negative or no influence was found in EM patients (Figures 1C and D).

\section{Correlation Analyses}

The correlation analyses showed a positive correlation between migraine frequency and causal effect from the left hippocampus to $\mathrm{mPFC}$ across all migraine patients $(\mathrm{r}=0.573, \mathrm{P}<0.001)$ (Figure 4A). In addition, 

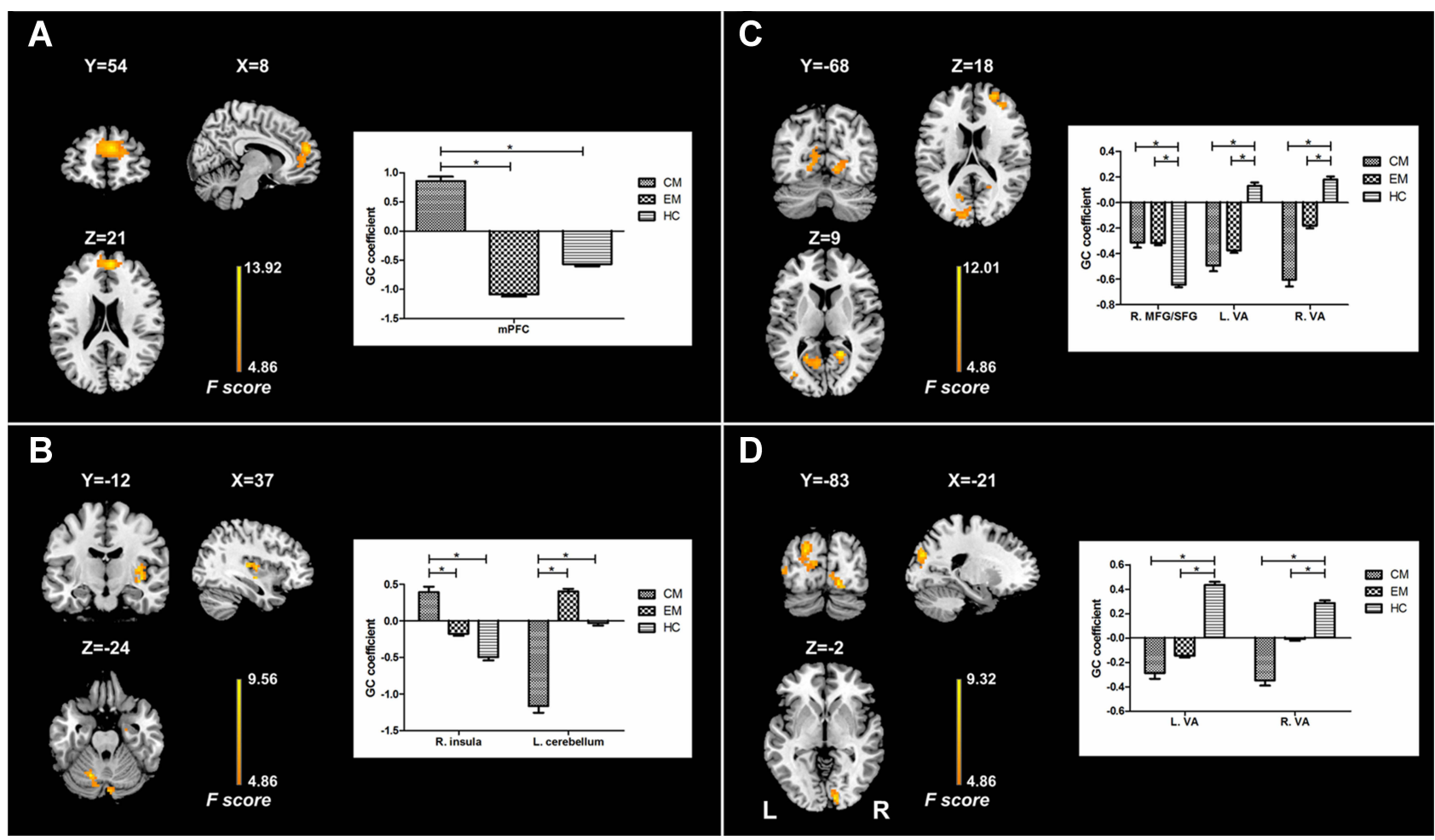

Figure I Results of analyses of variance for effective connectivity of the left and right hippocampus. The left panel shows regions with significant difference in causal flow from the left hippocampus (A) and right hippocampus (B) to other regions as well as extracted GC coefficients for these regions. The right panel shows regions with significant difference in causal flow from other regions to the left hippocampus (C) and right hippocampus (D) as well as extracted GC coefficients for these regions. Note: *Indicates significant difference between groups.

Abbreviations: GC, Granger causality; mPFC, medial prefrontal cortex; MFG, middle frontal gyrus; SFG, superior frontal gyrus; VA, visual areas; EM, the episodic migraine group; CM, the chronic migraine group; HC, the healthy control group.

migraine frequency was negatively correlated with causal effect from the right hippocampus to left cerebellum $(\mathrm{r}=0.488, \mathrm{P}<0.001)$ (Figure 4B). No significant correlation was found between other migraine-related clinical variables and causal flow from or to the hippocampus.

\section{Discussion}

This study examined the directed connectivity of the hippocampus with the rest of the brain in migraine by employing Granger causality analysis. We found increased and decreased negative influence on the hippocampus exerted by the bilateral visual areas and right dorsolateral prefrontal cortex (dlPFC), respectively, in each migraine group compared with the $\mathrm{HC}$ group. The hippocampus showed increased positive influence on the right posterior insula and $\mathrm{mPFC}$, as well as increased negative influence on the left cerebellum in CM patients relative to EM patients and healthy controls. In addition, Granger causality coefficients in the mPFC and left cerebellum were correlated with migraine frequency across all migraine patients, suggesting that the EC alterations may reflect migraine symptom severity.

The dlPFC is implicated in cognitive, affective, and sensory processing. ${ }^{25,26}$ Several lines of evidence support that the dlPFC acts as an interface between cognitive processing and pain regulation and can reduce pain via a cognitive control mechanism. ${ }^{27,28}$ Wiech et al. reported that dIPFC activity was negatively related to pain unpleasantness. ${ }^{27}$ Thus, reduced negative influence from the dIPFC to left hippocampus in the current study may suggest decreased inhibition and lead to exaggerated painrelated anxiety and unpleasantness. The absence of dIPFC difference between the migraine groups indicated that disrupted cognitive control may be common in patients with different migraine types. Supporting our result, abnormal brain structure and function of this region in both $\mathrm{CM}$ and EM patients have been reported in previous MRI studies on migraine. ${ }^{29-32}$

In both $\mathrm{CM}$ and $\mathrm{EM}$, the causal flow from the bilateral visual areas to bilateral hippocampus significantly decreased (increased negative influence), suggesting that 


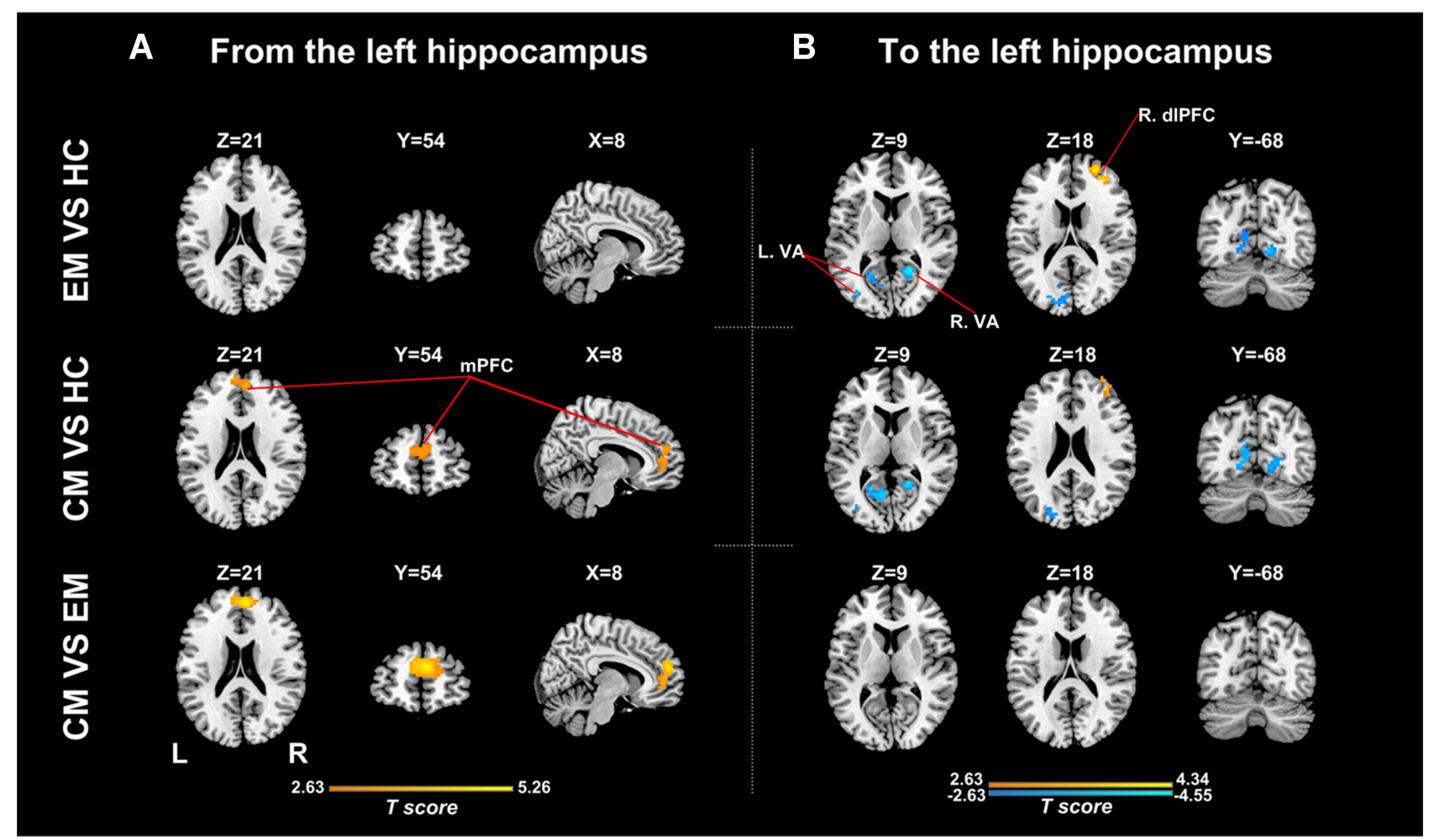

Figure 2 Between-group analyses for effective connectivity of the left hippocampus. (A) Regions showing significant between-group difference in causal flow from the left hippocampus to other regions; (B) Regions showing significant between-group difference in causal flow from other regions to the left hippocampus.

Abbreviations: EM, the episodic migraine group; CM, the chronic migraine group; HC, the healthy control group; mPFC, medial prefrontal cortex; dIPFC, dorsolateral prefrontal cortex; VA, visual areas.

disturbed EC between these two regions was independent of migraine frequency. The visual cortex is considered the main site for initiation of cortical spreading depression that heralds an ictal attack. ${ }^{33}$ Hyperexcitability and structural impairment was found in this region in the interictal state of patients with migraine with and without aura. ${ }^{32,34,35}$ Partially consistent with our results, atypical connectivity between the DMN regions and visual areas was reported in multiple lines of research on EM. ${ }^{36-38}$ However, the present study extended previous connectivity findings by delineating the direction of influence between the hippocampus and visual areas. Given the role of hippocampus in pain-related attention and anxiety, we speculated that increased negative influence from visual areas to right hippocampus may be a compensatory mechanism for maladaptive stress response in migraine.

Recently, mounting evidence has implicated that the cerebellum is engaged not only in motor control but also in cognitive and affective functions. ${ }^{39}$ The cerebellum, especially the posterior part, is thought to play an inhibitory role in pain perception. ${ }^{40,41}$ It is possible that increased negative influence from the hippocampus to left cerebellum in $\mathrm{CM}$ as revealed in the current study may aggravate the disruption of modulating involvement of the cerebellum. In accordance with our result, decreased gray matter volume in cerebellum was found in CM patients, ${ }^{42}$ and an association between clinical variable and gray matter volume in this region was observed in structural MRI study on $\mathrm{CM}^{43}$

It is of interest to find enhanced positive causal flow from hippocampus to $\mathrm{mPFC}$ in $\mathrm{CM}$, given that the $\mathrm{mPFC}$ is generally considered to mediate attenuation of pain perception via top-down cognitive control. Etkin et al. revealed that different subdivisions of mPFC may have different contributions to emotional processing. ${ }^{44}$ The dorsal part participates in appraisal and expression of negative emotion, whereas the ventral portion has a regulatory role with respect to limbic regions involved in generating emotional responses. ${ }^{44}$ Therefore, our data of increased positive influence to the dorsal mPFC from hippocampus may suggest a greater unpleasantness rating of headache pain in patients with CM. Intriguingly, a similar change in bottomup activation of mPFC by the limbic system was found in post-traumatic stress disorder, another disease involving 


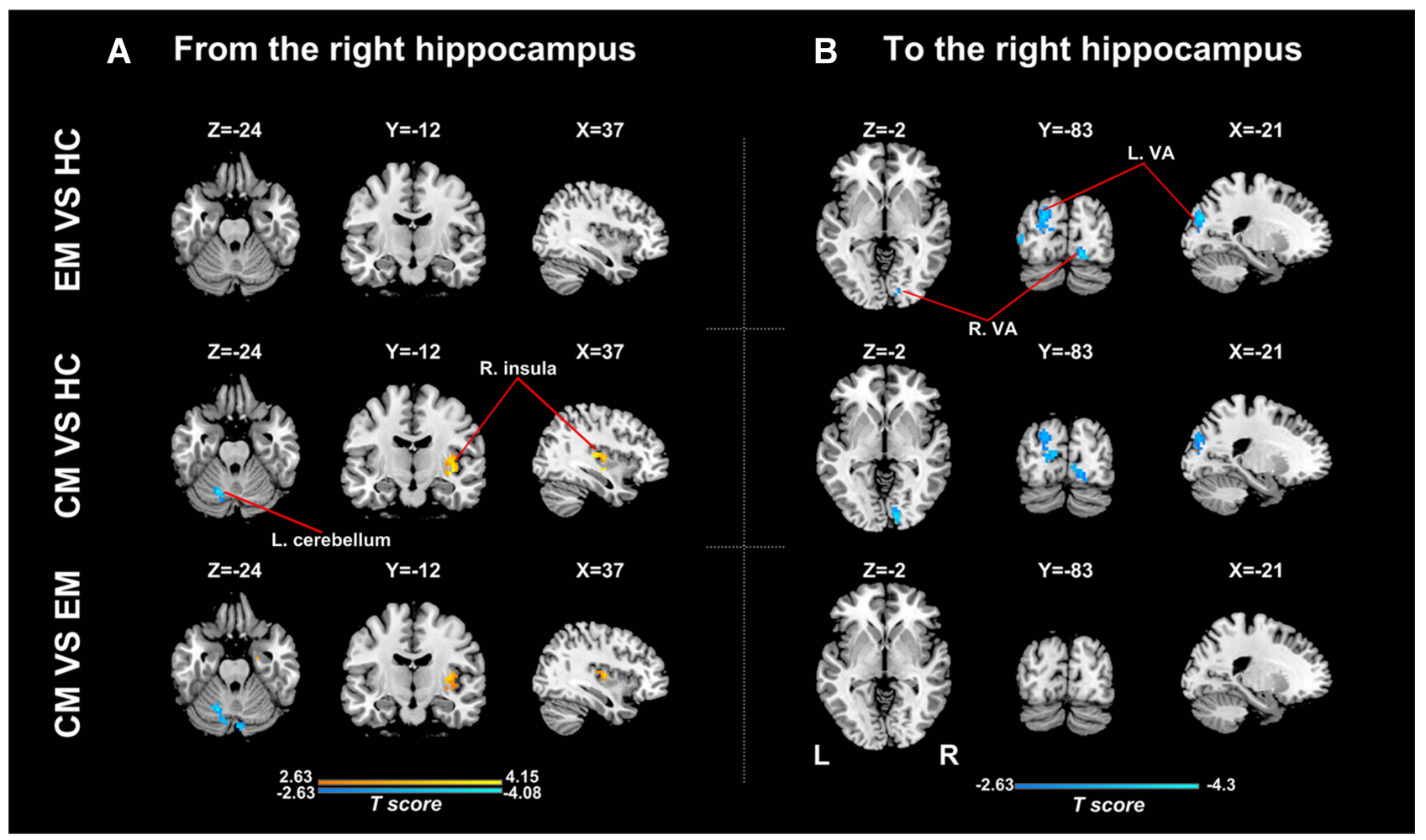

Figure 3 Between-group analyses for effective connectivity of the right hippocampus. (A) Regions showing significant between-group difference in causal flow from the right hippocampus to other regions; (B) Regions showing significant between-group difference in causal flow from other regions to the right hippocampus.

Abbreviations: EM, the episodic migraine group; CM, the chronic migraine group; HC, the healthy control group; VA, visual areas.

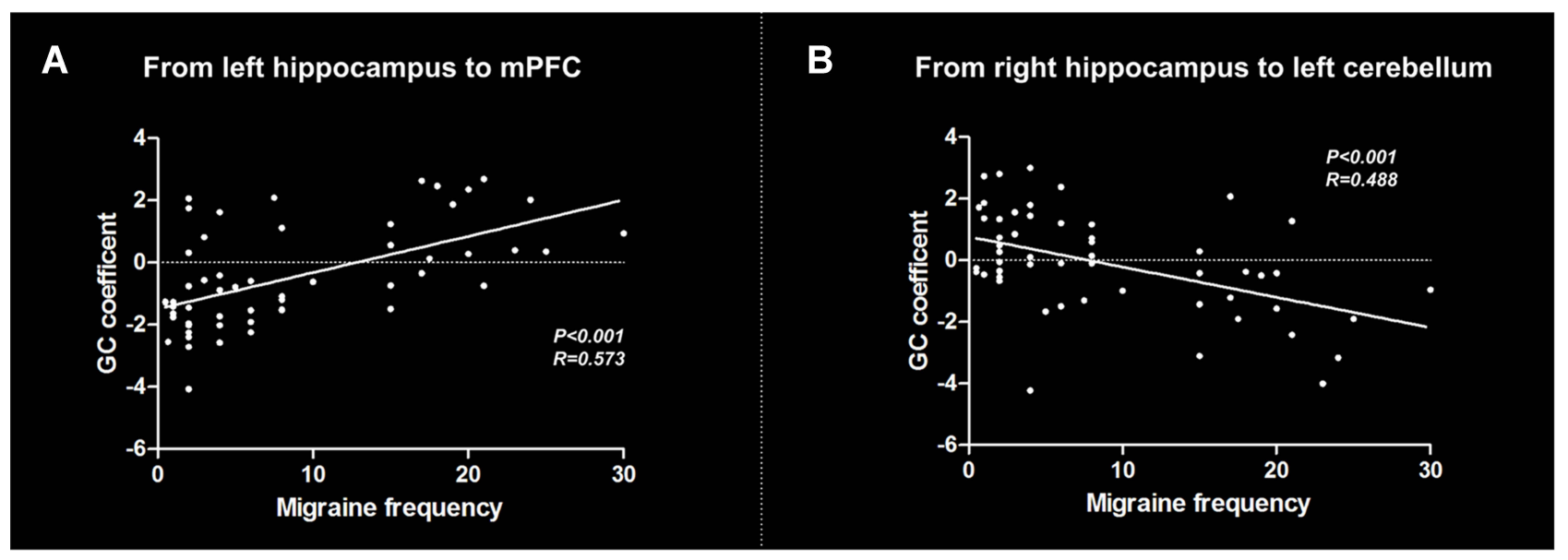

Figure 4 Results of correlation analyses between clinical variables and effective connectivity of the hippocampus across all migraine patients. (A) Correlation between migraine frequency and causal flow from the left hippocampus to mPFC; (B) Correlation between migraine frequency and causal flow from the right hippocampus to left cerebellum.

Abbreviations: GC, Granger causality; mPFC, medial prefrontal cortex.

a maladaptive stress response. $^{45}$ Moreover, across all patients in our study, the hippocampus-mPFC and hippocampus-cerebellum EC positively and negatively correlated with migraine frequency, respectively, which indicated that these imaging measures may be related to disease burden of migraine. This finding regarding $\mathrm{mPFC}$ was further supported by the result of analysis comparing the hippocampal EC in the FEM and IEM subgroups.

We also found increased positive influence from the right hippocampus to right posterior insula in the $\mathrm{CM}$ group compared with the other two groups. Right-sided lateralization of insula abnormalities has been observed in 
multiple neuroimaging studies on pain or migraine. ${ }^{46}$ As a region highly interconnected throughout the brain, the insula is involved in a wide range of processes, such as emotion, conscious awareness, autonomic regulation and sensation. ${ }^{47}$ The posterior insula is thought to play a more important role in sensorimotor integration than the anterior insula. Accordingly, our finding of greater positive influence from right hippocampus to posterior insula may suggest sensory hypersensitivity in CM patients. It is worth noting that functional and structural alterations were also reported in EM patients relative to healthy controls in prior literature. $^{38,48-50}$ The reason for no difference in EC between the hippocampus and insula in the EM versus $\mathrm{HC}$ comparison in the current study may be that the difference was too small to detect or that the abnormal hippocampus-insula EC was specific to $\mathrm{CM}$.

The present study had several limitations. First, since the sample size was small, the results should be considered preliminary. Second, the age and gender were not well balanced, though the difference for the latter was not statistically significant. Recent studies documented agerelated dysfunction in hippocampal FC and brain activity in healthy controls or EM patients. ${ }^{51,52}$ However, our findings were unlikely to be greatly influenced by age as no significant effect was found after including this variable as a covariate and some $\mathrm{EC}$ alterations in $\mathrm{CM}$ were further supported by the correlation analyses. Third, a comprehensive neuropsychological assessment was not performed to better define the impact of the disease. Finally, with a cross-sectional design, it could not be established whether the detected brain function abnormalities predispose a person to migraine or result from recurrent migraine attacks.

\section{Conclusion}

In summary, this study provides evidence for a key role played by the hippocampus in the pathophysiology of migraine. Migraine patients had disrupted EC between the hippocampus and multiple brain regions involved in the sensory processing and cognitive processing of pain. Altered negative influences on the hippocampus exerted by the dIPFC and visual areas were common characteristics for patients with EM and CM. In addition, directional influences from the hippocampus to $\mathrm{mPFC}$ and left cerebellum seem to be related to migraine chronicity and may act as useful imaging biomarkers for evaluating migraine frequency.

\section{Abbreviations}

mPFC, medial prefrontal cortex; dlPFC, dorsolateral prefrontal cortex; rs-fMRI, resting-state functional magnetic resonance imaging; FC, functional connectivity; DMN, default mode network; HC, healthy control; EM, episodic migraine; $\mathrm{CM}$, chronic migraine; $\mathrm{EC}$, effective connectivity; GCA, Granger causality analysis; ICHD, international classification of headache disorders; IEM, infrequent episodic migraine; FEM, frequent episodic migraine; VAS, visual analog scale; SPM, statistical parametric mapping; REST, Resting-State fMRI Data Analysis Toolkit; WM, white matter; CSF, cerebrospinal fluid; MNI, Montreal Neurological Institute; AAL, automated anatomical labeling; ANOVA, analysis of variance; DPABI, Data Processing and Analysis for Brain Imaging; MFG, middle frontal gyrus; SFG, superior frontal gyrus.

\section{Data Sharing Statement}

The raw data of this study are available from the corresponding author upon reasonable request.

\section{Ethical Approval and Informed Consent}

All procedures performed involving human participants were in accordance with the Declaration of Helsinki, and were approved by the ethics committee of the first affiliated hospital of Soochow University (Approval no. 2021246). Informed consent was acquired from all individual participants included in the study.

\section{Author Contributions}

All authors contributed to data analysis, drafting or revising the article, gave final approval of the version to be published, agreed to the submitted journal, and agree to be accountable for all aspects of the work.

\section{Funding}

This study was supported by the National Nature Science Foundation of China (grant number 81701669, 81971573), the Nature Science Foundation of Jiangsu Province (grant number BK20170368).

\section{Disclosure}

The authors declare that they have no conflicts of interest in this study. 


\section{References}

1. Arnold M. Headache classification committee of the International Headache Society (IHS) the international classification of headache disorders, 3rd edition. Cephalalgia. 2018;38(1):1-211. doi:10.1177/ 0333102417738202

2. Lipton RB, Bigal ME, Diamond M, Freitag F, Reed ML, Stewart WF. Migraine prevalence, disease burden, and the need for preventive therapy. Neurology. 2007;68(5):343-349. doi:10.1212/01.wnl.000025 2808.97649.21

3. Stovner LJ, Nichols E, Steiner TJ; GBD 2016 Headache collaborators. Global, regional, and national burden of migraine and tensiontype headache, 1990-2016: a systematic analysis for the Global Burden of Disease Study 2016. Lancet Neurol. 2018;17 (11):954-976. doi:10.1016/S1474-4422(18)30322-3

4. Buse DC, Manack A, Serrano D, Turkel C, Lipton RB. Sociodemographic and comorbidity profiles of chronic migraine and episodic migraine sufferers. J Neurol Neurosurg Psychiatry. 2010;81 (4):428-432. doi:10.1136/jnnp.2009.192492

5. Tolner EA, Chen SP, Eikermann-Haerter K. Current understanding of cortical structure and function in migraine. Cephalalgia. 2019;39 (13):1683-1699. doi:10.1177/0333102419840643

6. Schwedt TJ, Chiang -C-C, Chong CD, Dodick DW. Functional MRI of migraine. Lancet Neurol. 2015;14(1):81-91. doi:10.1016/S14744422(14)70193-0

7. Brennan KC, Pietrobon D. A systems neuroscience approach to migraine. Neuron. 2018;97(5):1004-1021. doi:10.1016/j.neuron.20 18.01 .029

8. Skorobogatykh K, Van Hoogstraten WS, Degan D, et al. Functional connectivity studies in migraine: what have we learned? J Headache Pain. 2019;20(1):108. doi:10.1186/s10194-019-1047-3

9. Buckner RL, Andrews-Hanna JR, Schacter DL. The brain's default network: anatomy, function, and relevance to disease. Ann NY Acad Sci. 2008;1124(1):1-38. doi:10.1196/annals. 1440.011

10. Liu MG, Chen J. Roles of the hippocampal formation in pain information processing. Neurosci Bull. 2009;25(5):237-266.

11. McEwen BS. Plasticity of the hippocampus: adaptation to chronic stress and allostatic load. Ann N Y Acad Sci. 2001;933(1):265-277. doi:10.1111/j.1749-6632.2001.tb05830.x

12. Maleki N, Becerra L, Borsook D. Migraine: maladaptive brain responses to stress. Headache. 2012;52(Supp12):102-106. doi:10. 1111/j.1526-4610.2012.02241.x

13. Chong CD, Dumkrieger GM, Schwedt TJ. Structural co-variance patterns in migraine: a cross-sectional study exploring the role of the hippocampus. Headache. 2017;57(10):1522-1531. doi:10.1111/ head.13193

14. Hubbard CS, Khan SA, Keaser ML, Mathur VA, Goyal M, Seminowicz DA. Altered brain structure and function correlate with disease severity and pain catastrophizing in migraine patients. eNeuro. 2014;1(1):e20 14. doi:10.1523/ENEURO.0006-14.2014

15. Liu J, Lan L, Li G, et al. Migraine-related gray matter and white matter changes at a 1-year follow-up evaluation. J Pain. 2013;14 (12):1703-1708. doi:10.1016/j.jpain.2013.08.013

16. Liu J, Lan $\mathrm{L}, \mathrm{Mu} J$, et al. Genetic contribution of catechol-O-methyltransferase in hippocampal structural and functional changes of female migraine sufferers. Hum Brain Mapp. 2015;36(5):1782-1795. doi:10.1002/hbm.22737

17. Yu Y, Zhao H, Dai L, et al. Headache frequency associates with brain microstructure changes in patients with migraine without aura. Brain Imaging Behav. 2021;15(1):60-67. doi:10.1007/s1168 2-019-00232-2

18. Gao Q, Xu F, Jiang C, et al. Decreased functional connectivity density in pain-related brain regions of female migraine patients without aura. Brain Res. 2016;1632:73-81. doi:10.1016/j.brainres. 2015.12.007
19. Tomasi D, Wang GJ, Volkow ND. Energetic cost of brain functional connectivity. Proc Natl Acad Sci USA. 2013;110(33):13642-13647. doi:10.1073/pnas. 1303346110

20. Maleki N, Becerra L, Brawn J, McEwen B, Burstein R, Borsook D. Common hippocampal structural and functional changes in migraine. Brain Struct Funct. 2013;218(4):903-912. doi:10.1007/s00429-0120437-y

21. Wei HL, Chen J, Chen YC, et al. Impaired functional connectivity of limbic system in migraine without aura. Brain Imaging Behav. 2020;14(5):1805-1814. doi:10.1007/s11682-019-00116-5

22. Kim DJ, Jassar H, Lim M, Nascimento TD, DaSilva AF. Dopaminergic regulation of reward system connectivity underpins pain and emotional suffering in migraine. J Pain Res. 2021;14: 631-643. doi:10.2147/JPR.S296540

23. Yuan K, Zhao L, Cheng P, et al. Altered structure and resting-state functional connectivity of the basal ganglia in migraine patients without aura. J Pain. 2013;14(8):836-844. doi:10.1016/j.jpain.20 13.02.010

24. DSouza AM, Abidin AZ, Leistritz L, Wismüller A. Exploring connectivity with large-scale granger causality on resting-state functional MRI. J Neurosci Methods. 2017;287:68-79. doi:10.1016/j.jneumeth. 2017.06.007

25. Raij TT, Numminen J, Närvänen S, Hiltunen J, Hari R. Strength of prefrontal activation predicts intensity of suggestion-induced pain. Hum Brain Mapp. 2009;30(9):2890-2897. doi:10.1002/hbm.20716

26. Seminowicz DA, Moayedi M. The dorsolateral prefrontal cortex in acute and chronic pain. J Pain. 2017;18(9):1027-1035. doi:10.1016/j. jpain.2017.03.008

27. Wiech K, Kalisch R, Weiskopf N, Pleger B, Stephan KE, Dolan RJ. Anterolateral prefrontal cortex mediates the analgesic effect of expected and perceived control over pain. $J$ Neurosci. 2006;26 (44):11501-11509. doi:10.1523/JNEUROSCI.2568-06.2006

28. Lorenz J, Minoshima S, Casey KL. Keeping pain out of mind: the role of the dorsolateral prefrontal cortex in pain modulation. Brain. 2003;126(Pt 5):1079-1091. doi:10.1093/brain/awg102

29. Lai TH, Chou KH, Fuh JL, et al. Gray matter changes related to medication overuse in patients with chronic migraine. Cephalalgia. 2016;36(14):1324-1333. doi:10.1177/0333102416630593

30. Qin Z, He XW, Zhang J, et al. Altered spontaneous activity and functional connectivity in the posterior pons of patients with migraine without aura. J Pain. 2020;21(3-4):347-354. doi:10.1016/j.jpain.20 19.08.001

31. Lim M, Jassar H, Kim DJ, Nascimento TD, DaSilva AF. Differential alteration of fMRI signal variability in the ascending trigeminal somatosensory and pain modulatory pathways in migraine. $J$ Headache Pain. 2021;22(1):4. doi:10.1186/s10194-020-01210-6

32. Magon S, May A, Stankewitz A, et al. Cortical abnormalities in episodic migraine: a multi-center 3T MRI study. Cephalalgia. 2019;39(5):665-673. doi:10.1177/0333102418795163

33. Woods RP, Iacoboni M, Mazziotta JC. Bilateral spreading cerebral hypoperfusion during spontaneous migraine headache. $N$ Engl J Med. 1994;331(25):1689-1692. doi:10.1056/NEJM199412223312505

34. Mulleners WM, Chronicle EP, Palmer JE, Koehler PJ, Vredeveld JW. Visual cortex excitability in migraine with and without aura. Headache. 2001;41(6):565-572. doi:10.1046/j.1526-4610.2001.041 006565.x

35. Palm-Meinders IH, Arkink EB, Koppen H, et al. Volumetric brain changes in migraineurs from the general population. Neurology. 2017;89(20):2066-2074. doi:10.1212/WNL.0000000000004640

36. Tu Y, Fu Z, Zeng F, et al. Abnormal thalamocortical network dynamics in migraine. Neurology. 2019;92(23):e2706-e2716. doi:10. 1212/WNL.0000000000007607

37. Niddam DM, Lai KL, Fuh JL, Chuang CY, Chen WT, Wang SJ. Reduced functional connectivity between salience and visual networks in migraine with aura. Cephalalgia. 2016;36(1):53-66. doi: $10.1177 / 0333102415583144$ 
38. Soheili-Nezhad S, Sedghi A, Schweser F, et al. Structural and functional reorganization of the brain in migraine without aura. Front Neurol. 2019;10:442. doi:10.3389/fneur.2019.00442

39. Timmann D, Drepper J, Frings M, et al. The human cerebellum contributes to motor, emotional and cognitive associative learning. Rev Cortex. 2010;46(7):845-857. doi:10.1016/j.cortex.2009.06.009

40. Stoodley CJ, Schmahmann JD. Evidence for topographic organization in the cerebellum of motor control versus cognitive and affective processing. Cortex. 2010;46(7):844. doi:10.1016/j.cortex.2009.11.008

41. Moulton EA, Schmahmann JD, Becerra L, Borsook D. The cerebellum and pain: passive integrator or active participator? Brain Res Rev 2010;65(1):14-27. doi:10.1016/j.brainresrev.2010.05.005

42. Bilgiç B, Kocaman G, Arslan AB, et al. Volumetric differences suggest involvement of cerebellum and brainstem in chronic migraine Cephalalgia. 2016;36(4):301-308. doi:10.1177/0333102415588328

43. Coppola G, Petolicchio B, Di Renzo A, et al. Cerebral gray matter volume in patients with chronic migraine: correlations with clinical features. J Headache Pain. 2017;18(1):115. doi:10.1186/s10194-0170825-Z

44. Etkin A, Egner T, Kalisch R. Emotional processing in anterior cingulate and medial prefrontal cortex. Trends Cogn Sci. 2011;15 (2):85-93. doi:10.1016/j.tics.2010.11.004

45. Gilboa A, Shalev AY, Laor L, et al. Functional connectivity of the prefrontal cortex and the amygdala in posttraumatic stress disorder. Biol Psychiatry. 2004;55(3):263-272. doi:10.1016/j.biopsych.2003.08.004
46. Ke J, Yu Y, Zhang X, et al. Functional alterations in the posterior insula and cerebellum in migraine without aura: a Resting-State MRI Study. Front Behav Neurosci. 2020;14:567588. doi:10.3389/fnbeh.20 20.567588

47. Borsook D, Veggeberg R, Erpelding N, et al. The insula: a "hub of activity" in migraine. Neuroscientist. 2016;22(6):632-652. doi:10. $1177 / 1073858415601369$

48. Li Z, Zeng F, Yin T, et al. Acupuncture modulates the abnormal brainstem activity in migraine without aura patients. Neuroimage Clin. 2017;15:367-375. doi:10.1016/j.nicl.2017.05.013

49. Lee J, Lin RL, Garcia RG, Kim J, Napadow V. Reduced insula habituation associated with amplification of trigeminal brainstem input in migraine. Cephalalgia. 2017;37(11):1026-1038. doi:10.11 77/0333102416665223

50. Maleki N, Linnman C, Brawn J, Burstein R, Becerra L, Borsook D. Her versus his migraine: multiple sex differences in brain function and structure. Brain. 2012;135(8):2546-2559. doi:10.1093/brain/ aws 175

51. Stark SM, Frithsen A, Stark CEL. Age-related alterations in functional connectivity along the longitudinal axis of the hippocampus and its subfields. Hippocampus. 2021;31(1):11-27. doi:10.1002/hipo. 23259

52. Lisicki M, D’Ostilio K, Coppola G, et al. Age related metabolic modifications in the migraine brain. Cephalalgia. 2019;39(8): 978-987. doi:10.1177/0333102419828984
Journal of Pain Research

\section{Publish your work in this journal}

The Journal of Pain Research is an international, peer reviewed, open access, online journal that welcomes laboratory and clinical findings in the fields of pain research and the prevention and management of pain. Original research, reviews, symposium reports, hypothesis formation and commentaries are all considered for publication. The manuscript management system is completely online and includes a very quick and fair peer-review system, which is all easy to use. Visit http:// www.dovepress.com/testimonials.php to read real quotes from published authors. 